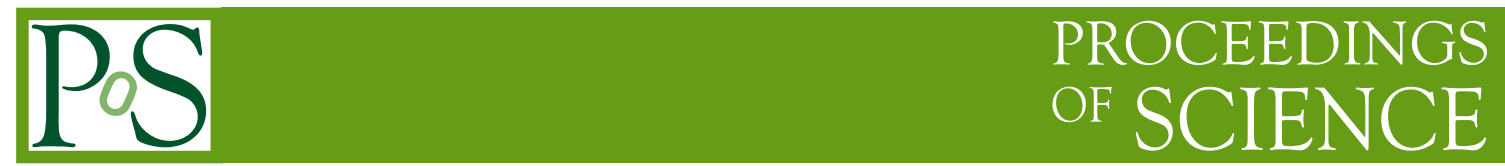

\title{
The dark side of inflation
}

\section{Gabriela Barenboim*}

Departamento de Fisica Teorica-IFIC, University of Valencia, Spain

E-mail: Gabriela.Barenboimeuv.es

\begin{abstract}
We present a new approach to quintessential inflation, in which both dark energy and inflation are explained by the evolution of a single scalar field. We start from a simple scalar potential with both oscillatory and exponential behavior.We employ the conventional reheating mechanism of new inflation, in which the scalar decays to light fermions with a decay width that is proportional to the scalar mass. Because our scalar mass is proportional to the Hubble rate, this gives adequate reheating at early times while shutting off at late times to preserve quintessence and satisfy nucleosynthesis constraints.
\end{abstract}

International Europhysics Conference on High Energy Physics

July 21st - 27th 2005

Lisboa, Portugal

${ }^{*}$ Research under grant FPA2002-00612 and GV05/267. 


\section{Introduction}

If we assume the correctness of the standard Friedmann equation evolution, the existence of dark energy engenders two profound dilemmas. The first is the cosmological constant problem, and the second is the "why now?" problem. Quintessence models $[1,2]$ attempt to address the these problems by introducing a very weakly coupled scalar field whose potential and/or kinetic function have special properties. In this spirit, we will describe a model with a simple scalar lagrangian with exponential and oscillatory features with only three adjustable parameters ${ }^{1}$ [4]. One parameter controls the period between infltionary epochs, a second controls the overall decay width, and the third parametrizes our ignorance about the relative fraction of matter versus radiation produced by reheating. These three parameters are adjusted to produce sufficient inflation along with the correct fractions $\Omega_{r} / \Omega_{\Lambda}, \Omega_{m} / \Omega_{\Lambda}$ of radiation, matter, and dark energy, as measured today. Having thus fixed the model we find that we automatically satisfy all constraints of primordial nucleosynthesis, $\mathrm{CMB}$, and large-scale structure.

\section{An oscillatory potential}

We start with a simple model with a single real scalar quintessence field $\theta$. The action is

$$
\int d^{4} x \sqrt{-g}\left[\frac{1}{2} f(\theta) g^{\mu v} \partial_{\mu} \theta \partial_{v} \theta-V(\theta)\right]
$$

where the kinetic function $f(\theta)$ and potential $V(\theta)$ are given by:

$$
f(\theta)=\frac{3 M_{\mathrm{P}}^{2}}{\pi b^{2}} \sin ^{2} \theta \quad ; \quad V(\theta)=\rho_{0} \cos ^{2} \theta \exp \left[\frac{3}{b}(2 \theta-\sin 2 \theta)\right],
$$

where $M_{\mathrm{P}}$ is the Planck mass: $1.22 \times 10^{19} \mathrm{GeV} ; \rho_{0}$ is the dark energy density observed today: $\simeq\left(10^{-4} \mathrm{eV}\right)^{4} ; b$ is a dimensionless parameter which controls the periodic behavior. A canonical kinetic term can be restored via a field redefinition $\theta(x) \rightarrow \phi(x)$, where $\phi(x)=\phi_{0} \cos \theta$ with $\phi_{0} \equiv \sqrt{3 M_{\mathrm{P}}^{2} / \pi b^{2}}$.

The expectation value of the quintessence field $\theta$ evolves logarithmically with scale factor from a positive initial value to zero today. Accelerated expansion corresponds to epochs (such as today) where $\theta$ is evolving through one of the fht "steps" of the potential. From (2.2) we see that the kinetic function is simultaneously suppressed in this epochs, slowing the roll of the scalar field evolution.

The equation of state parameter $w(a)$ has the same periodic form assumed in two recent phenomenological analyses [5, 6]. The analysis in [5] showed that, for $b=1, w(a)$ is consistent with all current data from observations of the CMB, Type IA supernovae, and large scale structure. It follows a fortiori that our model with any choice of $b$ less than one also agrees with this data.

To complete the model, we will assume that the quintessence field $\phi$ has a weak perturbative coupling to light fermions. This is the standard reheating mechanism of new inflation [3]. To avoid the strong constraints on long-range forces mediated by quintessence scalars [7], it is simplest to

\footnotetext{
${ }^{1}$ The model uses the conventional reheating mechanism of new inflation [3], in which the scalar decays to light fermions with a decay width that is proportional to the scalar mass.
} 


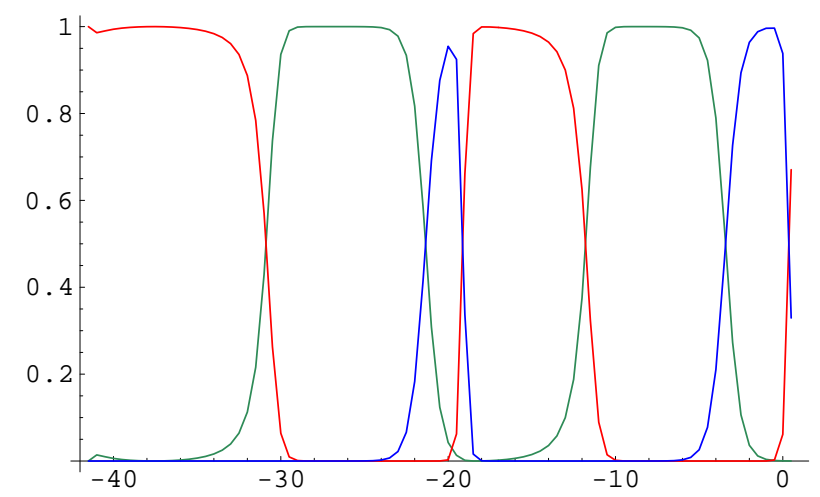

Figure 1: History of a slinky universe. Shown are the relative energy density fractions in radiation (green), matter (blue), and dark energy (red), as a function of the logarithm of the scale factor $a(t)$.

imagine that our scalar only has a direct coupling to a sterile neutrino. This is sufficient to hide the quintessence force from Standard Model nonsinglet particles [8], while still allowing the generation of a radiative thermal bath of Standard Model particles from quintessence decay.

With this assumption for reheating the evolution equations for quintessence, radiation, and matter become: $\dot{\rho}_{\theta}=-3 H(1+w) \rho_{\theta}-k_{0} m_{\phi}(1+w) \rho_{\theta} ; \dot{\rho}_{r}=-4 H \rho_{r}+\left(1-f_{m}\right) k_{0} m_{\phi}(1+w) \rho_{\theta}$ and $\dot{\rho}_{m}=-3 H \rho_{m}+f_{m} k_{0} m_{\phi}(1+w) \rho_{\theta}$ where $k_{0}$ and $f_{m}$ are small dimensionless constants. As long as $\theta$ is not near a multiple of $\pi / 2$, it is a reasonable approximation to make the replacement $k_{0} m_{\phi} \rightarrow k H$ where $k$ is another small dimensionless parameter. This replacement decouples the $\theta$ evolution equation from the Friedmann equation, giving an immediate analytic solution:

$$
\rho_{\theta}(a)=\rho_{0} \exp \left[\frac{1}{b}(3+k)(2 \theta-\sin 2 \theta)\right],
$$

We have used this approximation in the solutions quoted below.

\section{Results}

Figure 1 shows the results obtained from our model with $b=1 / 7, k=0.06$, and $f_{m}=10^{-11}$. Shown are the relative energy density fractions in dark energy, radiation, and matter, as a function of $\log a$. We have chosen to integrate the evolution equations starting from $a=10^{-42}$, which in our model corresponds to a temperature of slightly less than $10^{16} \mathrm{GeV}$, and an initial comoving Hubble radius of about 100 Planck lengths. For simplicity we have also chosen the value of $w(a)$ now to be exactly -1 . Neither of these choices corresponds to a necessary tuning.

Our three adjustable parameters have been chosen such that the values of $\Omega_{r} / \Omega_{\Lambda}, \Omega_{m} / \Omega_{\Lambda}$ come out to their measured values at $a=1$, and such that we have sufficient inflation. The latter is checked by computing the ratio of the fully inflated size of the intitial comoving Hubble radius to the current comoving Hubble radius. This ratio is about 3 in our model, indicating that the total amount of inflation is indeed enough to solve the horizon problem. The fhtness problem is solved because the total $\Omega_{r}+\Omega_{m}+\Omega_{\Lambda}=1$ within errors.

From Figure (1) we see that we are currently beginning the third epoch of accelerated expansion. The first epoch of inflation accumulated about $18 e$-foldings. Quantum fluctuations during 
this epoch produced the spatial inhomogeneities responsible for large scale structure and CMB anisotropies observed today. Constraints on the physics responsible for the primodial power spectrum of these density fluctuations can be set with WMAP and $2 \mathrm{dF}$ data, under the assumption that the Hubble rate is dominated by the contribution from $\rho_{\phi}$ during the observable part of inflation [9].

To compare with contraints from primordial nucleosynthesis [11], we note that the second epoch of dark energy domination ended well before $a \simeq 10^{-10}$, the time at which nucleosynthesis occured. Indeed dark energy reheating effects are completely negligible from $a \simeq 10^{-10}$ until today.

Figure (1) also shows one (very brief) prior epoch of matter domination. This only occurs if there is some suitably heavy and long-lived matter around to go out of thermal equilibrium when $a \sim 10^{-22}$, e.g. a superheavy neutrino [10].

It is fair to say that, compared to the simple model presented above, the standard new inflation scenario looks rather extreme. In the evolution history portrayed in Figure 1, the interplay between inflationary expansion and reheating is much milder. In fact, apart from a few very brief periods, reheating effects in our model do not actually increase the temperature of the thermal radiation bath. Instead, the temperature is almost always decreasing, but it decreases more slowly than in the standard $\Lambda$ CDM evolution.

Our particular realization of Slinky inflation must be regarded as a toy model (pun intended), since $\phi_{0}>M_{\mathrm{P}}$ and we have not invoked any consistent Planck scale framework such as string theory. However the model itself is surprisingly simple, and the physical picture which emerges from it has some compelling features. These are worthy of further investigation. Also, since the target space parametrized by our scalar is $\mathbf{S}^{\mathbf{1}}$, it would be interesting to extend this scenario to a class of nonlinear sigma models with other compact target spaces.

\section{References}

[1] S. Dodelson, M. Kaplinghat and E. Stewart, Phys. Rev. Lett. 85, 5276 (2000) [arXiv:astro-ph/0002360].

[2] P.J.E. Peebles \& B. Ratra, Phys. Rev. D37 3406 (1988); I. Zlatev, L. Wang, \& P.J. Steinhardt, Phys. Rev. Lett. 82, 896 (1998); P.J. Steinhardt, L. Wang, \& I. Zlatev, Phys. Rev. D59, 123504 (1999); A. Albrecht \& C. Skordis, astro-ph/9908085 (1999).

[3] L. F. Abbott, E. Farhi and M. B. Wise, Phys. Lett. B 117, 29 (1982).

[4] G. Barenboim and J. D. Lykken, arXiv:astro-ph/0504090.

[5] G. Barenboim, O. Mena and C. Quigg, arXiv:astro-ph/0412010.

[6] B. Feng, M. Li, Y. S. Piao and X. Zhang, arXiv:astro-ph/0407432.

[7] S. M. Carroll, Phys. Rev. Lett. 81, 3067 (1998) [arXiv:astro-ph/9806099].

[8] R. Fardon, A. E. Nelson and N. Weiner, JCAP 0410, 005 (2004) [arXiv:astro-ph/0309800].

[9] D. J. Schwarz and C. A. Terrero-Escalante, JCAP 0408, 003 (2004) [arXiv:hep-ph/0403129].

[10] G. Barenboim, L. Borissov, J. D. Lykken and A. Y. Smirnov, JHEP 0210, 001 (2002) [arXiv:hep-ph/0108199].

[11] R. Bean, S. H. Hansen and A. Melchiorri, Nucl. Phys. Proc. Suppl. 110, 167 (2002) [arXiv:astro-ph/0201127]. 